\title{
Combustion of mixtures containing the activated aluminium powder
}

\author{
Yana Dubkova ${ }^{1, *}$, Andrei Mostovchikov ${ }^{2}$, and Valery Kuznetsov ${ }^{1}$ \\ ${ }^{1}$ National Research Tomsk State University, 634050 Tomsk, Russia \\ ${ }^{2}$ National Research Tomsk Polytechnic University, 634050 Tomsk, Russia
}

\begin{abstract}
The ballistic characteristics of the mixture compositions containing aluminum powder ASD-6M have been studied. It is shown that the activation of aluminum powder by microwave radiation leads to an increase in the combustion heat of the mixture composition by $11 \%$.
\end{abstract}

\section{Introduction}

Nanoscale and micron powders of metals are increasingly used in various fields of engineering, chemical and metallurgical industries, medicine, metal-working manufacturing, etc. Also, metal powders are widely used in power engineering: they are used as an additive that increases the energy of combustion of high-energy materials. Thus, an increase in the stored energy in the added metal powder will allow the intensification of the combustion of high-energy material. It has now been established that the effect of accelerated electron flows [1], as well as microwave radiation [2], makes it possible to increase the specific thermal effect of oxidation of micro- and nanopowder metals. Thus, it is relevant to study the effect of the irradiation of a metal powder on the energy of combustion of a high-energy material that has such a modified powder in its composition.

The purpose of this work is to study the combustion rate and c heat of combustion of high-energy materials containing in their composition the micron powder of aluminum type ASD-6M, both untreated and processed by microwave radiation.

\section{Materials and methods of the experimental research}

To conduct experiments on the activation of aluminum powder, microwave electromagnetic radiation with a carrier frequency of $2.8 \mathrm{GHz}$, a pulse repetition frequency of $25 \mathrm{~Hz}$, and a pulse duration of $25 \mathrm{~ns}$ was used. Scheme experimental stand for irradiation experiments powders previously described in [2].

Pre-measured the initial dispersion and the activated alumina powder microwaves ASD-6M laser diffraction instrument "Mastersizer 2000".

The efficiency of the microwave activation of ASD-6M powder was determined using the differential thermal analysis method on the STD Q600 instrument to change the value of the exothermal effect of aluminum oxidation when a sample weighing $5 \mathrm{mg}$ was heated

\footnotetext{
* Corresponding author: y.a.dubkova@niipmm.tsu.ru
} 
in air at a rate of $10{ }^{\circ} \mathrm{C} / \mathrm{min}$. The error in determining the values of the thermal effects was $\pm 1.8 \%$.

To conduct studies on the calorific value and the burning rate, mixtures were prepared consists the basis of ammonium perchlorate and divinyl rubber containing $15 \mathrm{wt} . \%$ of the investigated aluminum powder.

After curing the samples, tests were conducted to determine the burning rate in air at atmospheric pressure and tests to determine the calorific value of combustion in a calorimeter bomb. All studies to determine the calorific value were carried out in accordance with the procedure described in $[3,4]$.

\section{Experimental results}

Results of powder analysis of ASD-6M and ASD-6M SHF (super-high frequencies) powders are presented in the form of histograms of the particle size distribution in Fig. 1 and in the form of a table (Table 1). It can be noted that the average volume diameter D (4.3) of the particles of the activated aluminum powder is $22 \%$ less than the original one. The ASD-6M microwave powder differs from the ASD-6M powder with a more developed surface and a narrower distribution width. Table 1 shows that the difference between the ASD-6M and ASD-6M microwave powders in D (0.50) is $19.5 \%$, in D (3.2) about $18.3 \%$, $\mathrm{D}(4.3)$ is $21.7 \%$.

Table 1. Key data of ASD-6M and ASD-6M SHF powders dispersion analysis.

\begin{tabular}{|c|c|c|}
\hline \multirow{2}{*}{ Characteristic, $\boldsymbol{\mu m}$} & \multicolumn{2}{|c|}{ Type } \\
\cline { 2 - 3 } & ASD-6M & ASD-6M (SHF) \\
\hline $\mathrm{D}(0.00)$ & 0.42 & 0.42 \\
\hline $\mathrm{D}(0.10)$ & 1.95 & 1.58 \\
\hline $\mathrm{D}(0.50)$ & 4.19 & 3.37 \\
\hline $\mathrm{D}(0.90)$ & 7.82 & 6.31 \\
\hline $\mathrm{D}(0.100)$ & 24.50 & 11.53 \\
\hline $\mathrm{D}_{10}$, mean diameter & 1.14 & 1110 \\
\hline $\mathrm{D}_{32}$, average surface diameter & 3.32 & 2.71 \\
\hline $\mathrm{D}_{43}$, average volume diameter & 4.74 & 3.71 \\
\hline
\end{tabular}

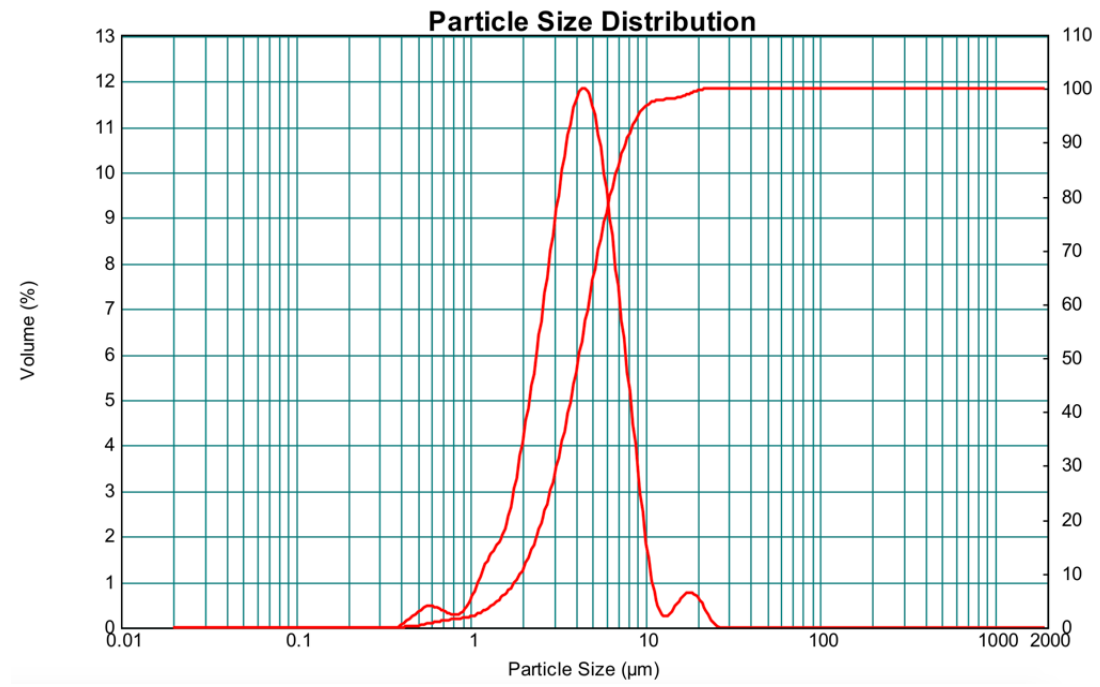

(a) 


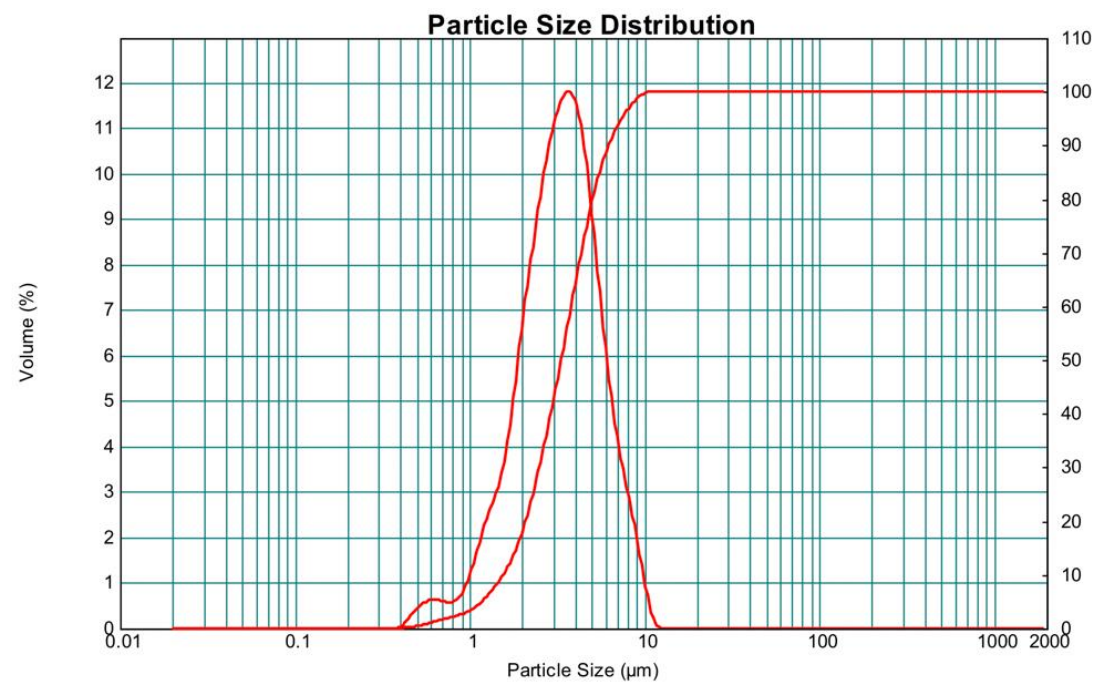

(b)

Fig. 1. Histogram of the distribution of aluminum powder particles: (a) ASD-6M, (b) ASD-6M(SHF).

Fig. 2 shows thermograms of thermal decomposition of aluminum nanopowder before (a) and after irradiation (b). When comparing these thermograms, it is seen that in the irradiated powder, the mass gain is less by $3.1 \%$ than in the irradiated powder. At the same time, in the irradiated aluminum powder, the specific thermal oxidation effect (total for two stages) increased by $780 \mathrm{~J} / \mathrm{g}(\sim 10.3 \%)$. In addition, the specific thermal effect of aluminum melting in micron powder decreased by $\sim 9.9 \%$. Taking into account that the degree of oxidation slightly decreased after irradiation, the increase in the heat of oxidation and the decrease in the heat of fusion can be explained by the additional allocation of the energy of radiation stored by the powder. Thus, this additional stored energy of radiation can be used to increase the energy of oxidation of mixed fuel compositions.

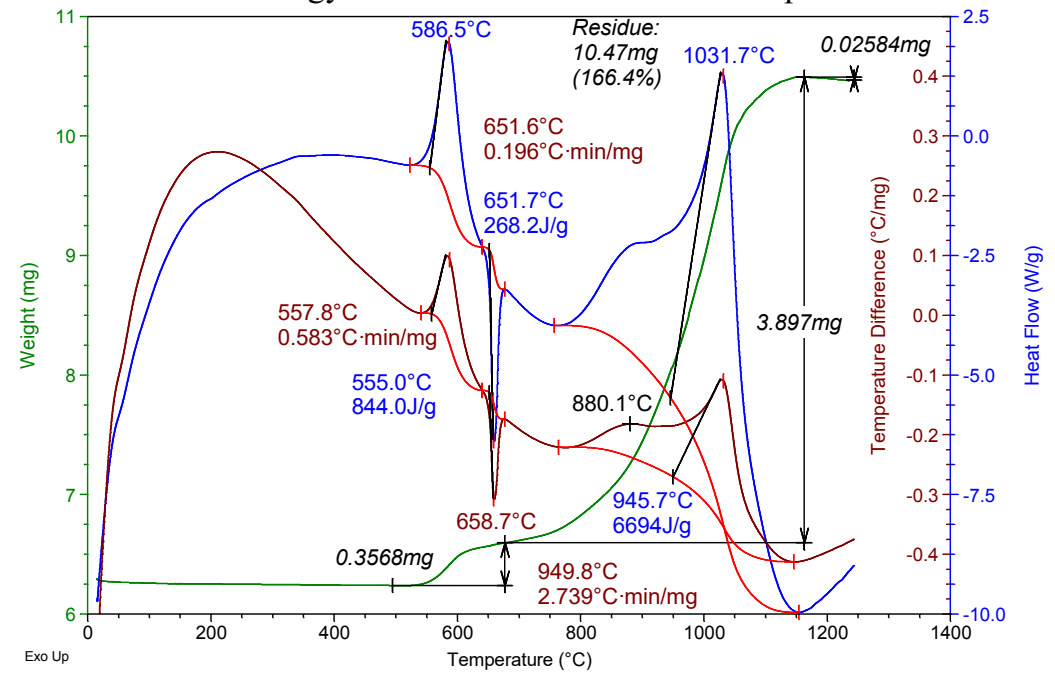

(a) 


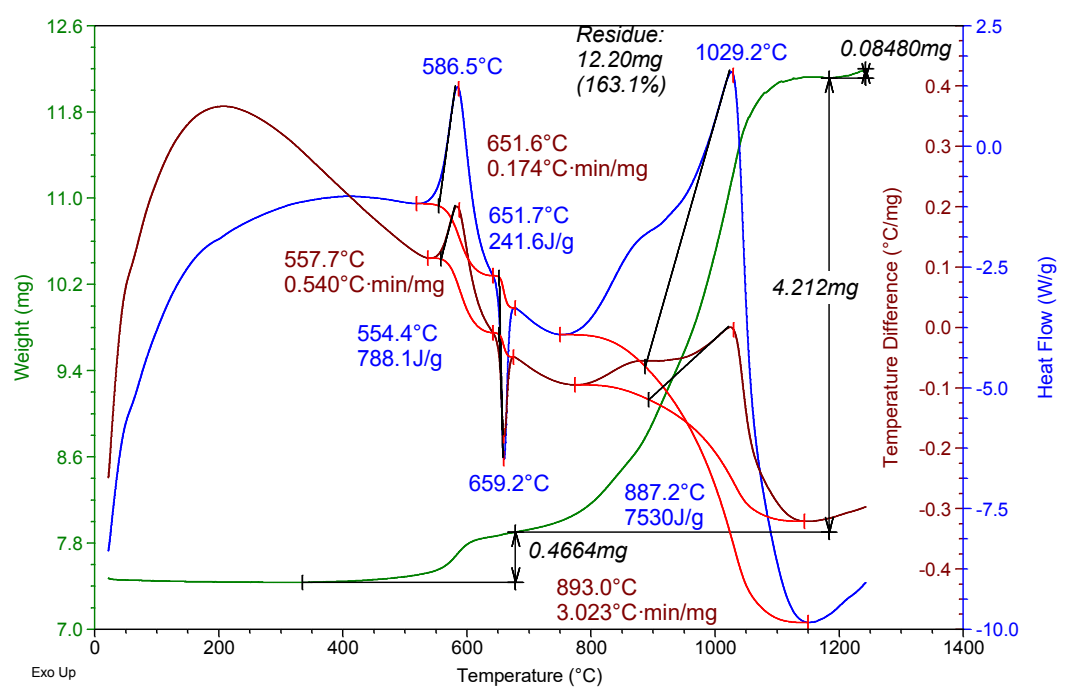

(b)

Fig. 2. Aluminum powders thermograms: (a) ASD-6M, (b) ASD-6M (SHF).

During the studies of heat and combustion rate, it was experimentally established that the linear combustion rate of samples containing $15 \%$ aluminum powder is practically independent of the type of powder (initial and activated by microwave radiation). However, the heat of combustion of the blended composition containing the activated aluminum powder is $11 \%$ higher than the heat of combustion of the composition with the original aluminum powder.

A summary of the study results o is presented in Table 2 .

Table 2. The results of the investigation of aluminum powder and compositions based on it.

\begin{tabular}{|c|c|c|c|c|c|}
\hline \multicolumn{4}{|c|}{ Aluminum powder characteristics } & \multicolumn{2}{c|}{ Mixtures characteristics } \\
\hline $\begin{array}{c}\text { Powder } \\
\text { type }\end{array}$ & $\begin{array}{c}\text { Particle } \\
\text { size, } D(4.3)\end{array}$ & $\begin{array}{c}\text { Oxidation onset } \\
\text { temperature, } \\
T_{\text {o.t. }}{ }^{\circ} \mathrm{C}\end{array}$ & $\begin{array}{c}\text { Oxidation } \\
\text { thermicity, } \\
\Delta \mathrm{H}, \mathrm{J} / \mathrm{g}\end{array}$ & $\begin{array}{c}\text { Burning rate, } \\
\mathrm{mm} / \mathrm{s}\end{array}$ & $\begin{array}{c}\text { Heat of } \\
\text { combustion, } \\
\mathrm{MJ} / \mathrm{kg}\end{array}$ \\
\hline ASD-6M & 4.74 & $\sim 540$ & 7270 & $1.57 \pm 0.04$ & $5.60 \pm 0.1$ \\
\hline $\begin{array}{c}\text { ASD-6M } \\
\text { SHF }\end{array}$ & 3.71 & $\sim 435$ & 8076 & $1.52 \pm 0.03$ & $6.22 \pm 0.1$ \\
\hline
\end{tabular}

\section{Conclusion}

Thus, the conducted studies showed that the activation of aluminum powder ASD-6M by exposure to microwave radiation, leads to a change in its dispersion and an increase in the heat of combustion of the mixture compositions containing activated aluminum. It was found that activation of aluminum powder by microwave radiation increases its dispersion, and the average volume diameter of the particles is reduced by $22 \%$. The oxidation temperature of the activated powder decreases by more than $100{ }^{\circ} \mathrm{C}$, and the total thermal oxidation effect increases by $11 \%$. Replacing the batch of industrial aluminum powder with the activated microwave radiation practically did not affect the value of the linear combustion rate of the mixture composition. When combusted in a calorimeter bomb, samples of fuel with activated aluminum are separated by $11 \%$ more heat compared to 
samples containing non-activated aluminum powder. Thus, the obtained results indicate that activation of aluminum powder ASD-6M with the help of microwave radiation increases heat release during oxidation of aluminum under non-isothermal conditions and during combustion in the composition of the mixture composition.

This work was financially supported by the Ministry of Education and Science of the Russian Federation, Project No. 11.1928.2017/4.6 and by the Ministry of Education and Science of the Russian Federation in terms of state order, project code 9.9036.2017.

\section{References}

1. A. P. Il'in, L. O. Root, A. V. Mostovshchikov, Tech. Phys. 57, 1178 (2012)

2. A. V. Mostovshchikov, A. P. Il'in, P. Yu. Chumerin, Yu. G. Yushkov, V. A. Vaulin, B. A. Alekseev, Tech. Phys. Letters, 42, 344 (2016)

3. Ya. Dubkova, A. Zhukov, MATEC Web Conf. 92, 01068 (2017)

4. S. Basalaev, A. Usanina, Ya. Dubkova, MATEC Web Conf. 110, 01022 (2017) 